\title{
The Distribution of the RR Lyrae Variables in $\omega$ Centauri and Background Light
}

\author{
J. O. Petersen ${ }^{1,2}$ and M. Andersen ${ }^{2}$ \\ ${ }^{1}$ Theoretical Astrophysics Center, and \\ ${ }^{2}$ Niels Bohr Institute for Astronomy, Physics and Geophysics, \\ Astronomical Observatory, Øster Voldgade 3, DK 1350 Copenhagen K, \\ Denmark, (E-mail: oz@astro.ku.dk and mia@astro.ku.dk)
}

\begin{abstract}
The distribution of the RR Lyrae stars in the globular cluster $\omega$ Centauri is studied, using the data published by Martin in 1938. Comparing the accumulated numbers of variables within the angular distance, $r$, from the centre of the cluster, it is shown that the known RRc variables seem to be less concentrated towards the centre than the RRab stars. 6-10 RRc stars are missing within $r=2-3$ arcmin, if the two distributions are actually identical, as expected from evolution models. We conclude that the central region may contain several RRc stars that have not yet been discovered. This could be due to their relatively low amplitude, since it is very difficult to find low-amplitude variables by means of photographic photometry in the crowded, central region of $\omega$ Cen with large and varying corrections due to unresolved background light.
\end{abstract}

\section{Introduction}

According to the standard scenario for the evolution of stars in the horizontal branch in globular clusters (e.g., Lee et al. 1990) the basic physical properties of the fundamental mode RRab variables and the first overtone RRc stars are identical, except for the well known difference in effective temperature. Since most globular clusters contain less than dozen or so RR Lyrae variables, they cannot provide data that can be used to compare the distributions of RRab and RRc variables. A significant test clearly requires a large number of both RRab and RRc stars, and only a few clusters are rich in both types of variables. $\omega$ Centauri contains at least about 80 RRab variables and $60 \mathrm{RRc}$ stars, and Martin (1938) published very detailed data for most of these variables. Here we use Martin's data collected in Petersen (1994) to discuss the distribution of the RR Lyrae variables in $\omega$ Cen.

\section{Radial distribution of RRab and RRc stars}

For each variable in his table, Martin (1938) gives the angular distance, $r$, of the star from the centre of the cluster. Martin analyses the effect of unresolved background light on his photographic magnitudes. He concludes that these effects are significant in a central region of $r \approx 4 \operatorname{arcmin}$. We have studied the 
distribution of RRab and RRc variables including 74 RRab and 55 RRc stars with data taken from Petersen (1994). It is interesting to compare the RRc distribution with the RRab data multiplied by $55 / 74$. We find that systematic differences are present. For $r \leq$ about 9 arcmin the number of RRc stars is 6-10 less than the number expected from the RRab distribution, whereas the number of RRc stars seems to be a little too high from $r=11$ arcmin to about 15 arcmin. It is tempting to explain the difference between the RRc distribution and the predicted numbers by the hypothesis that 6-10 RRc stars are missing within the inner 2 arcmin of the cluster. This is precisely the crowded core region, where Martin finds large systematic corrections to his photographic magnitudes.

\section{Conclusion}

The natural conclusion from this comparison of the distribution of RRab and $\mathrm{RRc}$ variables in $\omega$ Cen is that about $8 \mathrm{RRc}$ stars are actually present within the central 2 arcmin in $\omega$ Cen, although they have not been identified until now. Of course there could also be as yet undiscovered RRab stars present in the central region of $\omega$ Cen. If that should turn out to be the case, the expected number of "new" RRc stars should be increased with the additional number of RRab variables.

Martin (1938) could estimate the systematic effects of unresolved background light in $\omega$ Cen on his photographic magnitudes. He found that these effects are significant, typically $0.05-0.20 \mathrm{mag}$, within the central 4 arcmin. Besides the systematic corrections, background light will also result in additional statistical errors on photographic magnitudes, because the influence of the background (and crowding in the fields) will vary from one exposure to another, e.g., due to seeing. Clearly, it is more difficult to discover low-amplitude RRc variables here than in the outer regions of $\omega$ Cen. Therefore it may not be surprising, if the photographic surveys that have been used until now, have not succeeded in identifying several RR Lyrae variables - in particular low-amplitude RRc stars - in the central region of globular clusters. Modern CCD-based photometry will be able to provide much more accurate photometry in fields with background light and crowding than traditional photographic photometry.

Acknowledgments. We wish to thank Henning E. Jørgensen for many discussions on variable stars. Support from the Danish National Research Foundation via TAC is gratefully acknowledged.

\section{References}

Lee, Y.-W., Demarque, P., \& Zinn, R. 1990, ApJ, 350, 155

Martin, W.C. 1938, Leiden Annalen, Vol. XVII, Part 2

Petersen, J.O. 1994, A\&AS, 105, 145 